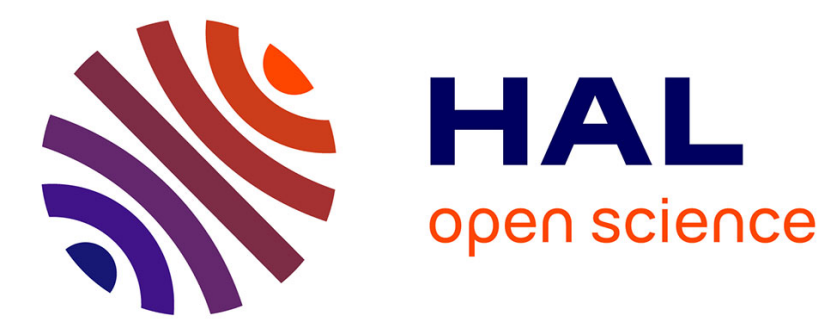

\title{
Les méfaits des guerres sur les forêts du canton de Munster (Haut-Rhin) \\ Daniel Doll
}

\section{To cite this version:}

Daniel Doll. Les méfaits des guerres sur les forêts du canton de Munster (Haut-Rhin). Revue forestière française, 1986, 38 (2), pp.165-171. 10.4267/2042/25634 . hal-03423757

\section{HAL Id: hal-03423757 \\ https://hal.science/hal-03423757}

Submitted on 10 Nov 2021

HAL is a multi-disciplinary open access archive for the deposit and dissemination of scientific research documents, whether they are published or not. The documents may come from teaching and research institutions in France or abroad, or from public or private research centers.
L'archive ouverte pluridisciplinaire HAL, est destinée au dépôt et à la diffusion de documents scientifiques de niveau recherche, publiés ou non, émanant des établissements d'enseignement et de recherche français ou étrangers, des laboratoires publics ou privés. 


\title{
LES MÉFAITS DES GUERRES SUR LES FORÊTS \\ DU CANTON DE MUNSTER (HAUT-RHIN) (*)
}

\author{
D. DOLL
}

Plus que tout autre élément du paysage, la forêt reflète et immortalise les méfaits de l'histoire. Les guerres successives (1914-1918 et 1939-1945), par leur violence et leurs effets prolongés, marquent profondément les surfaces boisées de la vallée de Munster à l'ouest de Colmar. Les dégâts, énormes autour des zones d'affrontements, affectent également une bonne partie des peuplements à l'arrière des fronts, touchès par la mitraille, l'occupation militaire, les incendies et les invasions de parasites. Pour les communes concernées, les conséquences financières sont fortes, et les cantons forestiers profondément atteints prennent une physionomie nouvelle qui confère au paysage son cachet actuel.

\section{LA FORÊT MUNSTÉRIENNE LIEU D'AFFRONTEMENTS VIOLENTS}

Le passage de la "guerre de mouvement " à la guerre des tranchées, en 1915, et la stabilisation du front dans la vallée, jouent un tour funeste à la forêt du canton. Les combats acharnés au Linge-Schratzmaennele (au nord du village d'Hohrod), à l'llienkopf (près de Metzeral), au col du Sattel (permettant la communication entre grande et petite vallée) et sur la crête entre le Hilsenfirst (Sondernach) et le Petit Ballon, mutilent de nombreux cantons forestiers. Si l'on excepte les affrontements de la basse vallée, près de Wihr-au-Val, et les escarmouches au Hohneck et dans l'arrière vallon de Mittlach, la forêt munstérienne a été, par contre, relativement épargnée par les combats de la Seconde Guerre mondiale.

\section{Le no man's land}

La ponction sylvicole, autour des zones de combat de la Guerre de 1914-18 est énorme. Six forêts communales sont particulièrement touchées: Breitenbach, Munster, Soultzeren, Sondernach, Muhlbach et Hohrod (cf. carte). La forêt de Hohrod est de loin la plus éprouvée par la guerre. Situées au sud de la crête culminante du Linge, les parcelles du vallon de Gunsbach qui forment la première ligne allemande, sont le siège d'incessants combats de 1914 à l'armistice. Sur les versants s'échelonnent les batteries allemandes d'obusiers et lance-mines; le sol est sillonné de boyaux, fouillé d'abris profonds, couvert de nappes de réseaux de deuxième ligne.

(") Un article du même auteur sur "L'évolution des essences forestières dans le canton de Munster (1850-1984) " a été publie dans la Revue forestière française, $n^{\circ} 6,1985, \mathrm{pp} .491-500$. 


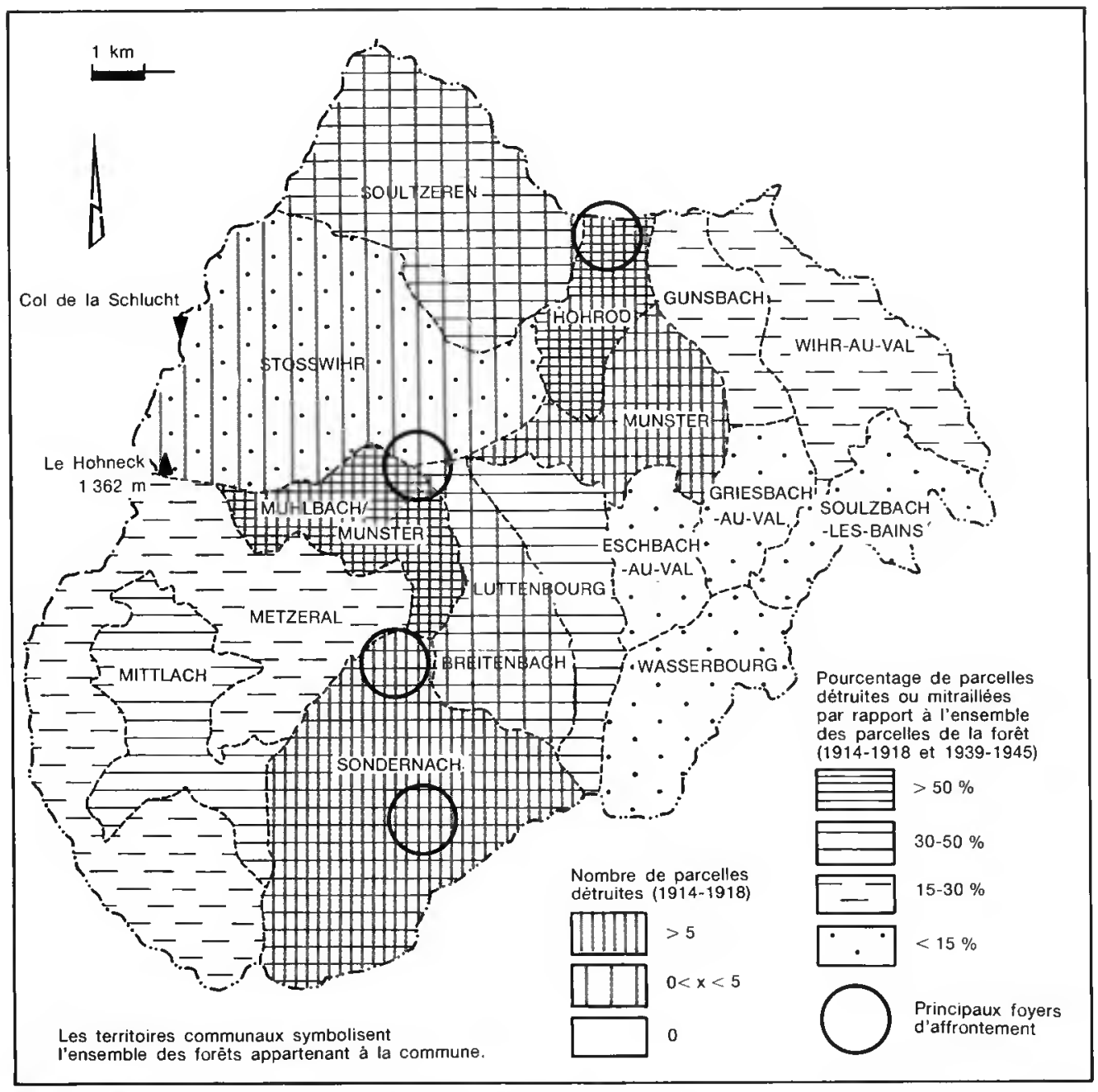

MÉFAITS OE LA GUERRE SUR LES FORÊTS COMMUNALES DU CANTON.

Source: d'après chiffres P.V.A.

En 1920, l'ètude des dommages de guerre estime la surface dètruite à 118 hectares (10 parcelles sur 24) et à 53 hectares la surface endommagée. Les experts relèvent $16,8 \mathrm{~km}$ de tranchées et de boyaux, $123000 \mathrm{~m}^{2}$ de réseaux et 168 abris de premier ordre. A Sondernach, autre no man's land, 9 parcelles sont fortement abîmées et une quinzaine sont touchẻes par la mitraille. $\dot{A}$ la suite de bombardements répétés au Hilsenfirst, au Hittstatt et au Breitstein, il ne reste qu'une masse de bois secs et brisés exploitée pour le chauffage à proximité du village. Dans ces endroits, la forêt fait place à des zones dénudées rapidement envahies par une abondante végètation de Sureaux, de Framboisiers et d'Épilobes. Dans les parties supérieures, où les dommages sont encore plus grands, le forestier est obligé d'abandonner les bois abattus à la pourriture, sans pouvoir dégager les semis qui les entourent. Les forêts de Muhlbach (100 ha détruits au Sattelkopf, Klitzerstein, Altmattkopf, Waldle, llienkopf, Reichsackerkopf), de Munster (147 ha abîmės au Mönchberg. Sattel, Silberwald et Frauenackerkopf) et de Soultzeren (23 parcelles touchées, destruction de Hörnleskopf et du Mülwenwald) connaissent un sort analogue et complètent le dramatique tableau. 


\section{Forêts mitraillées et dégradées}

Les dégâts ne se limitent pas, malheureusement, à la zone des affrontements. Derrière le front, une bonne partie des peuplements forestiers souffre des bombardements et de l'occupation militaire. La mitraille, extrêmement disséminée dans tout le canton, cause de graves blessures à de nombreux sujets. Seules les forêts de Griesbach et de Soultzbach en sont presque totalement dépourvues. L'organisation de position de batteries et d'abris divers, l'ouverture de routes stratégiques et de lignes de force électrique, et l'installation de chemins de fer à voie étroite (forêt de Wihr-au-Val), sont responsables de bien des dégâts. Contrairement aux Allemands soucieux de maintenir le couvert de la zone avancée, les troupes françaises multiplient les coupes stratégiques pendant la Première Guerre mondiale pour dégager les vues sur la plaine de Colmar. Au sommet du Zwergberg, trois hectares sont entièrement rasés.

La perturbation des cantons forestiers épargnés par les combats est aussi le résultat d'exploitations militaires abusives et anarchiques. Dans les parcelles 19 et 22 en forêt de Wihr-au-Val, quantité de jeunes hêtres et chênes sont coupés pour la carbonisation; les taillis de l'Estenbach servent à la confection de centaines de piquets de réseaux. Les dommages causés par les exploitations militaires sont plus limités dans les forêts du fond de la grande vallée, à l'intérieur des lignes françaises (Mittlach, Metzeral); les agents forestiers de l'administration des Eaux et Forêts en Alsace se chargent de les diriger et de les contrôler. La Seconde Guerre mondiale, en revanche, n'épargne pas ces forêts. Les Allemands, à la recherche de bois moyens, facilement exploitables, les trouvent en dehors de l'affectation à régénérer et désorganisent ainsi l'ensemble des surfaces boisées. Au lendemain des deux guerres mondiales, la forêt est dans un état pitoyable.

\section{LES EFFETS INDUITS DE LA GUERRE}

La guerre poursuit ses effets néfastes au-delà de la période belliqueuse. Par suite d'étés particulièrement secs, quelques incendies se déclarent et plusieurs espèces de Scolytes envahissent les forêts du canton (1921-26, 1947-49).

\section{L'incendie de Hohrod}

Le plus important incendie, celui de Hohrod, est provoqué, le 13 avril 1921, par la destruction d'un dépôt de grenades, au-dessus du col du Bärenstall. Poussé par un vent d'est et trouvant un aliment exceptionnel dans une forêt littéralement hachée par les obus, où les bois de toutes dimensions, cassés ou renversés, s'empilent sur plusieurs mètres d'épaisseur, recouvrant d'innombrables obus non éclatés, le feu, d'une violence inouie, dévore des arbres entiers, fait exploser obus, grenades et dépôts de munitions et parcourt 72 hectares dans la forêt de Hohrod et 19 hectares dans celle de Gunsbach. Le 15 avril au soir, l'incendie s'arrête. II ne reste guère, alors, dans le vallon de Gunsbach, que quelques bouquets de résineux à moitié brûlés (photos p. 168).

La série de cataclysmes n'est pas terminée pour autant, car au lendemain des guerres, profitant d'étés particulièrement chauds et secs, les insectes et leurs dégâts se multiplient.

\section{Les invasions de Scolytes}

Les peuplements misérables, abîmés par la Première Guerre mondiale, sont une proie facile pour le Bostryche liseré et le Bostryche typographe, qui trouvent dans les Épicéas blessés par les éclats d'obus d'excellents lieux de ponte. Pour combattre l'invasion, il aurait fallu résorber au 
plus vite l'excédent de bois mort, exploiter les arbres endommagés, écorcer les peuplements abattus aussitôt après exploitation et brûler l'écorce. L'abondance de bois mort et la pénurie de main d'œuvre ne permettent pas de mener à bien ces mesures d'assainissement et les ravages se poursuivent jusqu'en 1926. Cinq années d'invasions èprouvent fortement les cantons forestiers les plus affaiblis par le premier conflit mondial. Les «Bostryches" occasionnent de larges

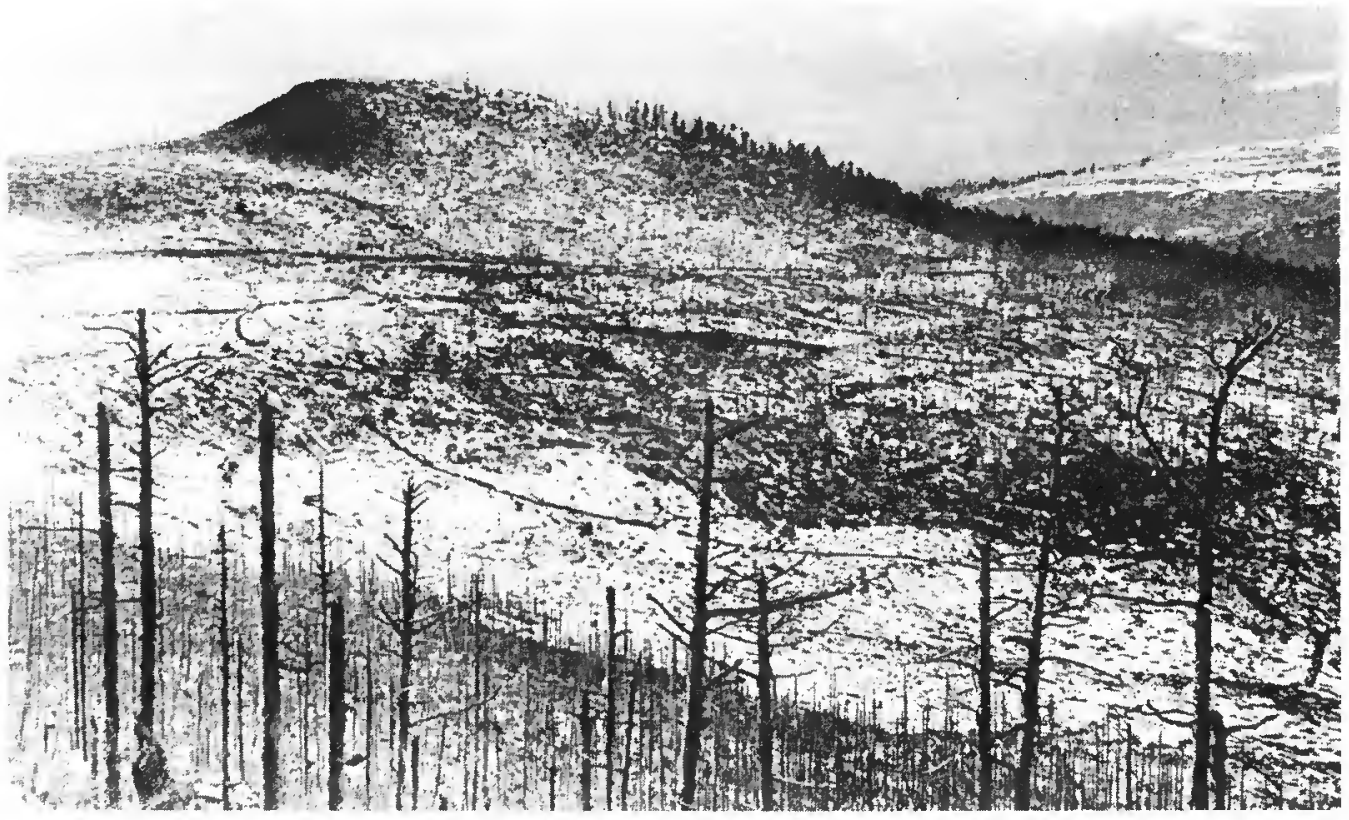

Le LINGE - Vue d'ensemble

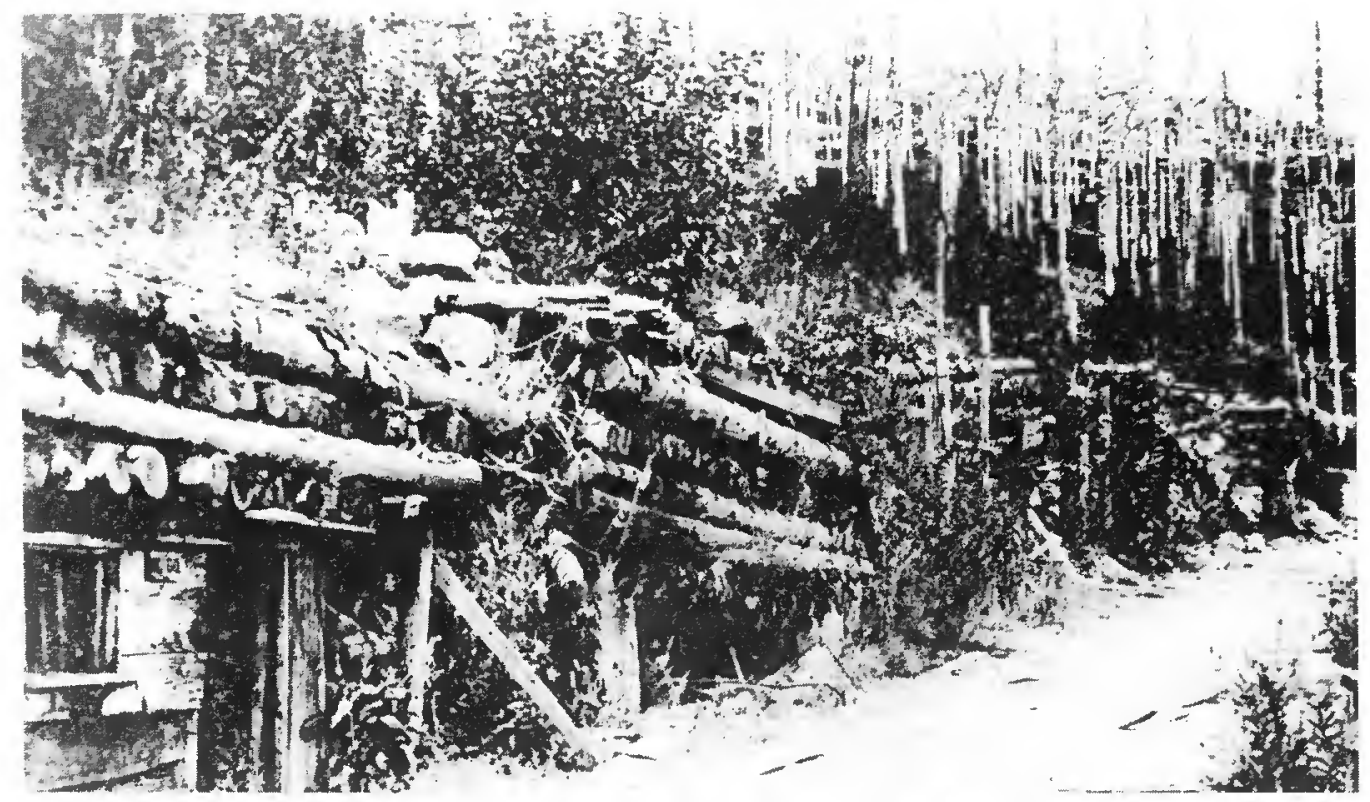

Environs d'ORBEY - Schratzmănnel - Abris allemands près du Linge 
vides qui s'ajoutent aux trouées de guerre. Les dégâts sont considérables à Munster, qui exploite $30000 \mathrm{~m}^{3}$ de bois bostrychés entre 1923 et 1935 . À Hohrod, après le passage des insectes, il ne reste plus rien en 1926 de la série en futaie régulière de l'avant-guerre. Dans la forêt de Stosswihr, où les surfaces détruites par le Bostryche (70 ha) sont quatre fois plus importantes que les dégâts de guerre (16 ha), les Coléoptères ne se contentent pas de s'attaquer aux peuplements affaiblis ou abîmés. Une étude par parcelles révèle une corrélation étroite entre invasion de Bostryche et monoculture d'Épicéas (tableau I).

Tableau I

Dégâts de Bostryche en forêt de Stosswihr (1922)

(source P.V.A. Stosswihr)

\begin{tabular}{|c|c|c|}
\hline Numéros de parcelles & $\begin{array}{c}\text { Surfaces détruites } \\
\text { par la guerre }\end{array}$ & $\begin{array}{c}\text { Surfaces dénudées } \\
\text { à la fin de l'année 1922 }\end{array}$ \\
\hline 5 & 1,5 ha & 3,5 ha \\
6 & 1,5 ha & 8,5 ha (100\% d'Epicéas) \\
7 & 2,5 ha & 3,5 ha \\
10 & 2 ha & 3,5 ha \\
15 & 1,3 ha & 9 ha (90\% d'Epicéas) \\
16 & 2 ha & 4 ha (90\% d'Epicéas) \\
17 & - & 10 ha (100\% d'Epicéas) \\
32 & - & 7 ha \\
\hline 35 & - &
\end{tabular}

$\mathrm{Au}$ lendemain de la Seconde Guerre mondiale, I'invasion de parasites xylophages en 1947 permet de confirmer ce qui aurait pu passer pour un hasard: les vigoureux résineux du vallon de Mittlach et de Wasserbourg, jusque là épargnés par les insectes, sont également victimes des "Bostryches". Dans la forêt de l'arrière vallée du Krebsbach, réputée pour sa résistance naturelle aux invasions d'insectes, Bostryches typographes et Bostryches curvidentés affectent profondément une bonne partie des Conifères. Seule la forêt domaniale du Herrenberg, composée d'essences mélangées ( $40 \%$ de Hêtre), sort indemne des deux invasions de Bostryches.

\section{LES CONSÉQUENCES : MODIFICATIONS PAYSAGẺRES ET PERTES FINANCIĖRES}

La réorganisation de la forêt s'impose après les terribles calamités qui se sont abattues sur elle. La solution retenue par le Service forestier haut-rhinois pour la reconstitution de ces forêts, est dictée autant par des considérations économiques que par des données géographiques et sylvicoles. Elle est à l'origine de la transformation des paysages forestiers. Mais aujourd'hui encore, il subsiste des bois mitraillés qui dévalorisent quelques forêts et engendrent de lourdes pertes financières pour les communes.

\section{L'enrésinement de la vallée par les essences non indigènes (1)}

Les cantons forestiers profondément endommagés par la guerre ne peuvent que difficilement s'intégrer à l'aménagement classique. Néanmoins, rares sont les forestiers décidés à mettre le champ de bataille hors aménagement et à laisser les peuplements au repos, pour permettre à la

(1)Épicea, Pin sylvestre et Douglas principalement. 
forêt de se refaire par ses propres moyens. À Munster, cette méthode donne pourtant des résultats positifs et la forêt se reconstitue partiellement d'elle-même. Un tel résultat n'a pu être acquis, il est vrai, que grâce à l'exposition nord de plusieurs cantons forestiers, à la qualité du sol et à l'existence de porte-graines d'essences de lumière. Au Mönchberg, où ces conditions ne sont pas remplies, des repeuplements artificiels s'avèrent nécessaires sur toute la surface. Dans les autres forêts du canton, des plantations massives d'Épicéa et, en nombre plus limité, de Pin sylvestre, de Mélèze et de Sapin, remplissent les trouées dues à la guerre. Le conflit de 1914-18 et, à un degré moindre celui de 1939-45, sont de puissants facteurs de l'enrésinement artificiel de la vallée par les essences allogènes.

De nombreux cantons forestiers prennent, de ce fait, une physionomie nouvelle. À Hohrod, où les forestiers plantent de jeunes résineux sur 170 hectares ( $57 \%$ de la superficie), la composition de la forêt se modifie considèrablement, comme en témoigne le tableau II. En 1957, les reconstitutions d'après guerre (1914-18) sont à peine terminées dans cette forêt.

Pour remettre les surfaces boisèes en ètat, les coupes portent principalement sur les arbres tarès et mitraillés, tandis que les parcelles en régénération sont laissées pour compte. Néanmoins, il n'est pas toujours facile de détecter les arbres minés par les éclats d'obus et les balles de fusil.

Tableau II Les effets de la guerre sur la composition des peuplements en forêt d'Hohrod (source P.V.A. Hohrod)

\begin{tabular}{|c|c|c|c|}
\hline \multirow{2}{*}{ Nature du peuplement } & \multicolumn{3}{|c|}{$\begin{array}{l}\text { Pourcentage des essences } \\
\text { par rapport à l'ensemble de la forêt }\end{array}$} \\
\hline & 1905 & 1957 & 1976 \\
\hline Sapin $\ldots \ldots \ldots \ldots \ldots \ldots \ldots \ldots \ldots \ldots$ & 65 & 23 & 21 \\
\hline Epicéa & 9 & 27 & 28 \\
\hline Pin sylvestre $\ldots \ldots \ldots$ & 11 & 34 & 34 \\
\hline Chêne . . & 7 & 8 & 6 \\
\hline Hêtre $\ldots \ldots \ldots \ldots \ldots \ldots$ & 8 & 4 & 3 \\
\hline Divers ..... & & 4 & 8 \\
\hline & $100 \%$ & $100 \%$ & $100 \%$ \\
\hline
\end{tabular}

\section{Les arbres mitraillés, un bois déprécié}

En raison de l'importance des surfaces mitraillées, il n'est pas possible de liquider le peuplement endommagé en quelques années. Or, un arbre visiblement mitraillé en 1920 est beaucoup moins décelable en 1930 et ne l'est plus du tout en 1940, comme le fait remarquer P. Gouttin [3] ${ }^{(2)}$.

De nos jours encore, on suppose qu'il subsiste un grand pourcentage de forêts suspectes de mitraille. Une reconnaissance effectuée par l'Office national des Forêts, portant sur neuf communes, évalue à près de 1518 le nombre d'hectares encore endommagés en 1960 (tableau III). Les arbres dépréciès occasionnent une forte baisse du prix d'achat et les scieurs, craignant de casser leurs lames de scie sur des éclats d'obus, refusent le bois issu des cantons forestiers supposés abîmés. Soucieux d'avoir du bois sain, quelques-uns d'entre eux vont jusqu'à s'approvisionner en Forêt-Noire.

(2)Les numèros entre crochets renvoient à la bibliographie en fin d'article. 
(source Office national des forêts de Colmar)

\begin{tabular}{|c|c|c|c|}
\hline Forêt communale & $\begin{array}{l}\text { Surface totale } \\
\text { en } 1960 \text { (en ha) }\end{array}$ & $\begin{array}{c}\text { Surface mitraillee } \\
\text { (en ha) }\end{array}$ & $\begin{array}{l}\text { Volume des bois } \\
\text { mitraillés (en } \mathrm{m}^{3} \text { ) }\end{array}$ \\
\hline Breitenbach & 537,28 & 56,0 & 13600 \\
\hline Hohrod ........ & 311,24 & 6,0 & 1800 \\
\hline Luttenbach ..... & 405,68 & 39,1 & 8000 \\
\hline Metzeral ....... & 955,77 & 169,5 & 52800 \\
\hline Muhlbach ..... & 708,25 & 162,5 & 50300 \\
\hline Munster ....... & 1749,25 & 285,6 & 84600 \\
\hline Sondernach $\ldots \ldots \ldots \ldots \ldots \ldots \ldots \ldots . \ldots \ldots$ & 731,34 & 247,9 & 48100 \\
\hline Soultzeren...$\ldots \ldots \ldots \ldots \ldots \ldots \ldots$ & 907,90 & 463,0 & 105300 \\
\hline$\ldots \ldots \ldots \ldots \ldots$ & 992,23 & 88,4 & 24700 \\
\hline TOTAL & 7298,94 ha & 1518,0 ha & $389400 \mathrm{~m}^{3}$ \\
\hline
\end{tabular}

\section{CONCLUSIONS}

La reconstitution de la forêt après les ravages des guerres s'insère au début des années 1950 , dans un programme qui vise, sans oublier ses autres rôles, à rétablir son rôle "d'usine à bois" travaillant à plein rendement. Pour ce faire, les objectifs de production à réaliser prévoient la suppression des espaces improductifs et la levée, le plus rapidement possible, de l'hypothéque des bois mitraillés tout en réduisant les frais de récolte et de plantation. L'application de ces principes suppose la généralisation de pratiques forestières honnies jusque là (les coupes à blanc étoc dans les futaies et les plantations en complément de régénération) et l'adoption de véritables possibilités permettant l'élimination réelle des bois mitraillés [1]. L'assainissement jugé salutaire pour les communes, est également avantageux pour l'économie générale de l'aprèsguerre, car il met sur le marché un volume de bois accru, à une époque où, précisément, les résineux font défaut.

Les vicissitudes de l'histoire remettent en question l'idée genéralement répandue de l'inertie des paysages furestiers.

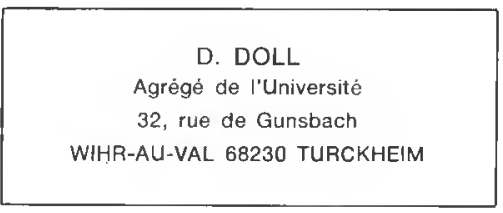

\section{BIBLIOGRAPHIE}

[1]BADRÉ (L.). - Évolution des aménagements des futaies résineuses dans l'Est de la France et plus spécialement dans le Haut-Rhin. - Revue forestiẻre française, $n^{\circ} 7-8,1952$, pp. 500-507.

[2]DOLL (D.). - Évolution du paysage forestier dans le canton de Munster. - T.E.R., Lyon II, 1983,175 pages.

[3]GOUTTIN (P.). - Les forêts mitraillées, soixante ans après. - Revue forestière française, $n^{\circ} 4$, 1979, pp. 318322.

[4]WEHRLEN (R.V.) - La promenade d'un bataillon d'instruction sur le tront de l'Est. - Édité par l'Amicale des Anciens du 21/42 R.I.F. Colmar, 1979.

[5]Les procès verbaux d'amenagements (P.V.A.) des forêts domaniales et communales du canton. 